\title{
Towards Programming Languages for Machine Learning and Data Mining (Extended Abstract)
}

\author{
Luc De Raedt and Siegfried Nijssen
}

Department of Computer Science, Katholieke Universiteit Leuven, Belgium

\begin{abstract}
Today there is only little support for developing software that incorporates a machine learning or a data mining component. To alleviate this situation, we propose to develop programming languages for machine learning and data mining. We also argue that such languages should be declarative and should be based on constraint programming modeling principles. In this way, one could declaratively specify the problem of machine learning or data mining problem of interest in a high-level modeling language and then translate it into a constraint satisfaction or optimization problem, which could then be solved using particular solvers. These ideas are illustrated on problems of constraint-based itemset and pattern set mining.
\end{abstract}

\section{Motivation}

Today, machine learning and data mining are popular and mature subfields of artificial intelligence. The former is concerned with programs that improve their performance on specific tasks over time with experience and the later one with analyzing data in order to discover interesting patterns, regularities or models in the data. The two are intimately related in that machine learning often analyses data in order to learn, and that data mining often employs machine learning techniques to compute the regularities of interest, which explains why we shall not always distinguish these two fields. Significant progress in the past few years has resulted in a thorough understanding of different problem settings and paradigms and has contributed many algorithms, techniques, and systems that have enabled the development of numerous applications in science as well as industry.

Despite this progress, developing software that learns from experience or analyzes data remains extremely challenging because there is only little support for the programmer. Current support is limited to the availability of some software libraries [21] and the existence of data mining tools such as Weka and Orange, most of which only support the most common tasks of machine learning and data mining. Using these libraries for a specific application requires at best a thorough understanding of the underlying machine learning principles and algorithms; at worst it is impossible because the tool does not directly support the targeted learning or mining task. What is lacking is a direct support for the programmer of the machine learning software. This has motivated Tom Mitchell 
to formulate the following long term research question in his influential essay The Discipline of Machine Learning [15]:

Can we design programming languages containing machine learning primitives? Can a new generation of computer programming languages directly support writing programs that learn? ... Why not design a new computer programming language that supports writing programs in which some subroutines are hand-coded while others are specified as to be learned?

This question is not really new; it represents an old but still unrealized dream that has been raised in a number different ways and contexts throughout the history of artificial intelligence. For instance, some adaptive programming languages $[1,20]$ have been developed that embed hierarchical reinforcement learning modules in programming languages, while probabilistic programming languages aim at integrating graphical models and uncertainty reasoning into programming languages $[9,4]$. Other endeavors related to this question concern inductive query languages, which extend database query languages with the ability to declaratively query for patterns and models that in a database; these patterns and models become 'first class citizens' and the idea is to tightly integrate data mining inside databases; this has been the topic of a lot of research since the introduction of the vision by Iemielinski and Mannila [13]; cf. [3], and automatic programming [2], inductive logic programming [16,5] and program synthesis by sketching [12] which all attempt to synthesize in one way or another programs from examples of their input and output behavior.

While all these approaches have contributed important new insights and techniques, we are still far away from programming languages and primitives that support the writing and integration of programs for machine learning problems that arise in many applications.

In this extended abstract, we outline some principles and ideas that should allow us to alleviate this situation and we illustrate them using a particular example taken from our work on combining itemset mining and constraint programming $[18,17]$. Using this preliminary work, some interesting directions for further research are pointed out.

\section{Machine Learning and Data Mining as Constraint Satisfaction and Optimization Problems}

What we believe is necessary to realize Mitchell's vision, is a way to declaratively specify what the underlying machine learning problem is rather than outlining how that solution should be computed. Thus a number of modeling and inference primitives should be provided that allow the programmer to declaratively specify machine learning and data mining problems. This should be much easier than implementing the algorithms that are needed to compute solutions to these problems. Contemporary approaches to machine learning and data mining are too procedural, that is, they focus too much on the algorithms and the optimizations that are necessary to obtain high performance on specific tasks and 
datasets. This makes it hard to identify common primitives and abstractions that are useful across a wide range of such algorithms. Yet abstraction is necessary to cope with the complexity of developing software. To make abstraction of the underlying algorithms we believe it is useful to assume that

\section{Machine learning and data mining tasks can be declaratively expressed as con-} straint satisfaction and optimisation problems.

This assumption can be justified by observing that it is common practice to define machine learning tasks as those of finding an approximation $\hat{f}$ of an unknown target function $f$ from data $D$ such that

1. $\hat{f}$ belongs to a particular hypothesis space $\mathcal{H}$, that is, $\hat{f} \in \mathcal{D}$;

2. $\hat{f}$ is a good approximation of the target function $f$ on the training data, that is, $\hat{f}(D) \approx f(D)$; and/or

3. $\hat{f}$ scores best with regard to a scoring function $\operatorname{score}(f, D)$, that is, $\hat{f}=$ $\arg \max _{f \in \mathcal{H}} \operatorname{score}(f, D)$.

This type of problem is essentially a constraint satisfaction and optimization problem where the requirements $\hat{f} \in \mathcal{D}$ and $\hat{f}(D) \approx f(D)$ impose constraints on the possible hypotheses and the second requirement $\hat{f}=\arg \max _{f \in \mathcal{H}} \operatorname{score}(f, D)$ involves the optimization step. In data mining, this is often formulated as computing a theory $T h(H, D, q)=\{f \in H \mid q(f, D)$ is true $\}$, where $H$ is the space of possibly hypotheses, $D$ the dataset and $q$ specifies the constraints and optimization criteria [14].

We shall refer to the ensemble of constraints and optimization criterion as the model of the learning task. Models are almost by definition declarative and it is useful to distinguish the constraint satisfaction problem, which is concerned with finding a solution that satisfies all the constraints in the model, from the optimization problem, where one also must guarantee that the found solution be optimal w.r.t. the optimization function. Examples of typical constraint satisfaction problems in our context include local pattern mining, where the constraints impose for instance a minimum frequency threshold, and concept-learning, where the hypothesis should be consistent w.r.t. all examples. Typical optimization problems include the learning of support vector machines, where one wants to minimize the loss, and the parameters of a graphical model, where one wants to maximize the likelihood.

\section{Declarative Programming for Machine Learning and Data Mining}

Specifying a machine learning or data mining problem as a constraint satisfaction and optimization problem enables us to treat machine learning and data mining problems as any other constraint satisfaction and optimization problem. General methodologies for solving wide ranges of constraint satisfaction problems, as well 
as the inclusion of these methodologies in programming languages, have been well studied within the field of constraint programming since the early 90s [19].

Applying the modeling principles of constraint programming to machine learning and data mining leads naturally to a layered approach in which one can distinguish:

the modeling (M) language is the most abstract language, which allows us to declaratively specify the problem; at this level the machine learning or data mining problem is encoded in a similar way as that used by machine learning and data mining researchers for specifying problems of interest;

the constraint satisfaction and optimization (CSO) language is a lower level language for specifying constraint satisfaction and optimization problems at an operational solver level, that is, at this level the problem is encoded in a way that is understood by solvers, and that may also include some procedural elements;

the programming $(\mathbf{P})$ language is the (traditional) programming language which serves as the host language; at this level one can outline how to compute the inputs for the machine learning and data mining models and how to process the outputs.

In constraint programming, Essence [6] is an example of a modeling language. It allows one to specify combinatorial problems in almost the same way at that used in textbooks (such as Garey and Johnsson's [7]). The challenge is to translate these specifications into models at the CSO level, so that solutions can be computed by existing solvers. Finally, these solvers are embedded inside traditional programming languages.

A benefit of this approach is that it decouples the modeling language from the solver. In this regard, not only constraint programming solvers, but also satisfiability solvers or integer programming solvers could be used as a backend, where applicable.

To illustrate these ideas, consider the following example pptaken from our work on combining constraint programming and pattern mining $[18,17]$, which fits within this paradigm. More specifically, we show the M and CSO-level specifications in Algorithms 1 and 2 for frequent itemset mining. This involves finding sets of items that frequently occur in a set of transactions. Frequent itemset mining is probably the simplest and best studied data mining problem.

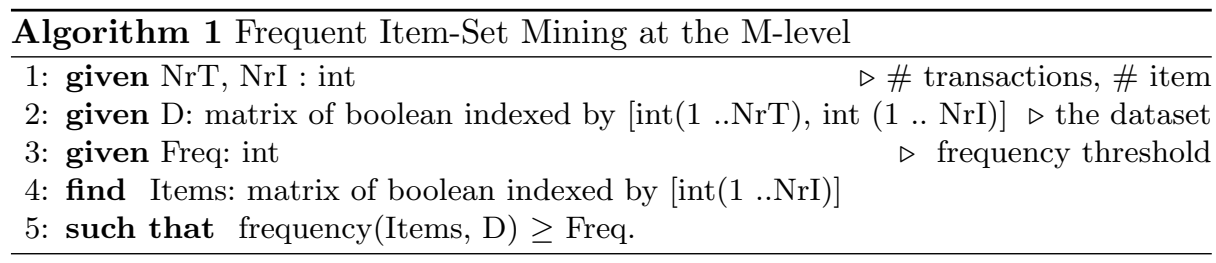


Algorithm 1 directly encodes the frequent itemset mining problem at the M-level. The CSO-level, illustrated in Algorithm 2, is a much more detailed level model which provides an efficient encoding that can almost directly be written down in the primitives supported by constraint programming systems such as Gecode [8]. ${ }^{1}$ In the CSO formulation of the problem, one searches for a combination of two vectors Items and Trans such that 1) the transaction-set encoded by Trans corresponds exactly to all transactions in the dataset that are covered by the itemset encoded by Items (the coverage constraint); and 2) the itemset is frequent; cf. $[18,17]$ for more details.

In a series of papers $[18,17]$ we have shown that the same declarative constraint programming principles can not only be applied to frequent itemset mining but also to a wide variety of constraint-based pattern mining tasks such as finding maximal, closed, discriminative itemsets, ... This often involves only minor changes to the constraints. For instance, finding maximal frequent itemsets involves changing the "=>" implication in the frequency constraint in a " $<=>$ " double implication, which shows the flexibility and power of the constraint programming approach. In addition, we have studied an extension of the pattern mining tasks to mining sets of $k$ patterns [10] that satisfy constraints and we have shown that several well-known data mining tasks such as concept-learning, tiling, redescription mining and a form of conceptual clustering can be modeled within this framework.

Compared to using pattern mining algorithms present in libraries such as Weka, the benefit of the proposed approach is that it is easier to extend with further constraints and that it is easier to extend towards other settings: whereas in the procedural approach it is necessary to modify the algorithm in the library itself, in our proposed approach it suffices to change the high-level model of the problem, where any constraint provided in the modeling language can be used. For instance, by including statistical tests as a primitive in a modeling language, we could easily extend frequent itemset mining towards finding statistically relevant itemsets without implementing a new algorithm [17].

\section{Challenges}

Whereas our previous work showed that constraint programming provides an interesting approach to addressing basic itemset mining problems, extending this approach towards other data mining and machine learning tasks faces several important challenges.

First, most current constraint programming systems are finite domain solvers, that is, they solve problems in which all variables are discrete and finite. In data mining and machine learning many tasks involve numerical computation and optimization, for instance, in statistical and Bayesian learning. Most current CP solvers are not well equipped to solve such numerical problems and do not yet

\footnotetext{
1 The notation used in Algorithm 2 deviates from the actual one used by Gecode, as Gecode models problems in the $\mathrm{C}++$ host language. It closely mirrors the way constraints CSO problems can be specified in Gecode, however.
} 


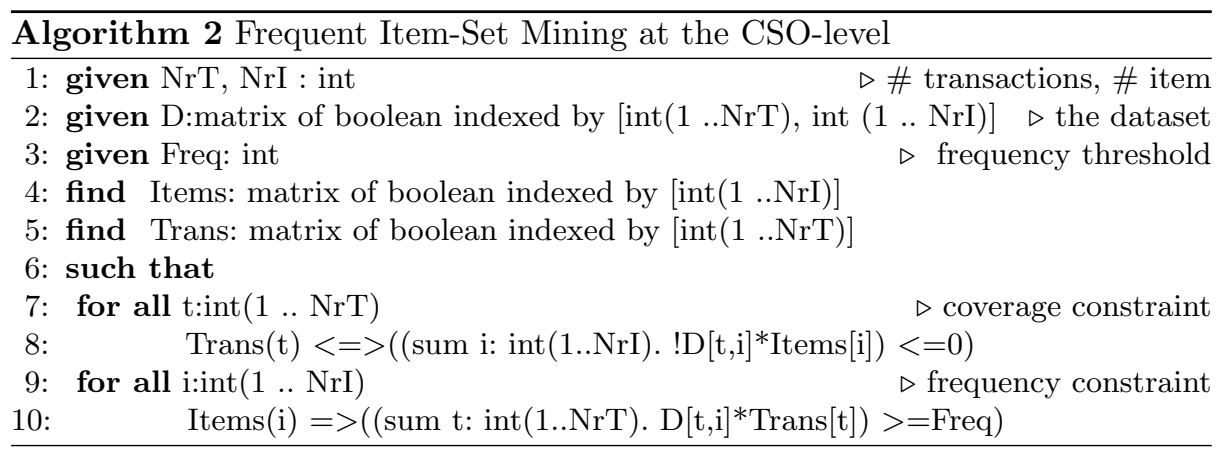

provide a suitable language at the CSO-level. To deal with this issue in a scalable and usable manner, solvers are needed that also support numerical primitives such as convex optimization.

Fortunately, a key feature of constraint programming systems is their extendibility towards new constraints. A core area of CP research is that of developing propagators for new global constraints, i.e. constraints that involve many variables. An initial step could be to develop global constraints, as well as their propagators, for a number of showcase applications in mining and learning, such as clustering and Bayesian modeling; initially, these constraints could operate on discrete decision variables, while later on adding non-discrete decision variables can be considered.

Second, many data mining and machine learning problems are computationally hard so that it cannot be expected that a global solution can be calculated in reasonable time. In many cases, this might not even be needed and finding a locally optimal solution is sufficient. A solver is needed in which the specified problem is solved using local search or heuristic search. Ideally this solver would make reasonable choices with respect to how to perform the local search by itself, and only limited input by the user is needed at the P-level. Also here programming languages for local search under development in the constraint programming community could provide a useful starting point [11].

Third, even in the discrete case the current CSO modeling languages are not well adapted to machine learning and data mining tasks. In any language for mining and learning one would expect support for basic concepts such as datasets, coverage, and error, but there is currently no language at the M-level which supports these and makes modeling easy. Developing a suitable language at the M-level cannot be seen independently from developments at the other levels. The M-level language should be such that automatically mapping it to an appropriate model at the CSO-level is feasible; if new primitives are needed in underlying solvers, their implementation should be made easy. Also at the Mlevel there are significant challenges in designing effective and declarative high level modeling primitives; for instance, to model statistical and Bayesian learning approaches. 
Fourth, a remaining challenge is that of dealing with structured data. When the data consists of networks, graphs, text, or logical descriptions, it is not clear how current solvers can be applied; it may be that new solvers are needed (CSOlevel), or that the mapping from the M-level to the CSO-level needs further study, exploiting results in grounding logical formulas, for instance.

\section{Conclusions}

In this extended abstract, it has been argued that programming languages for machine learning and data mining can be developed based on principles of constraint programming. This would involve declarative models specifying the machine learning or data mining problem at hand, and then, translating it into a lower level constraint satisfaction and optimization problem that can be solved using existing solvers. These preliminary ideas have been illustrated using our existing work on constraint programming and pattern mining. They leave open a large number of interesting research questions.

\section{Acknowledgements}

The authors would like to thank Tias Guns, Angelika Kimmig, and Guy Van den Broeck for interesting discussions about this work. Siegfried Nijssen is supported by the Research Foundation Flanders.

\section{References}

1. David Andre and Stuart J. Russell. Programmable reinforcement learning agents. In Todd K. Leen and Thomas G. Dietterich and Volker Tresp, editor, Advances in Neural Information Processing Systems, volume 13, pages 1019-1025, 2000.

2. A. Biermann, G. Guiho, and Y. Kodratoff, editors. Automatic Program Construction Techniques. Macmillan, 1984.

3. Jean-François Boulicaut, Luc De Raedt, and Heikki Mannila, editors. ConstraintBased Mining and Inductive Databases, European Workshop on Inductive Databases and Constraint Based Mining, Hinterzarten, Germany, March 11-13, 2004, Revised Selected Papers, volume 3848 of Lecture Notes in Computer Science. Springer, 2005.

4. L. De Raedt, P. Frasconi, K. Kersting, and S. Muggleton, editors. Probabilistic Inductive Logic Programming - Theory and Applications, volume 4911 of Lecture Notes in Artificial Intelligence. Springer, 2008.

5. Luc De Raedt. Logical and Relational Learning. Springer, 2008.

6. Alan M. Frisch, Warwick Harvey, Christopher Jefferson, Bernadette Martẃenez Hernández, and Ian Miguel. Essence : A constraint language for specifying combinatorial problems. Constraints, 13(3):268-306, 2008.

7. M. R. Garey and D. S. Johnson. Computers and Intractability: A Guide to the Theory of NP-completeness. Freeman, San Francisco, California, 1979.

8. Gecode Team. Gecode: Generic constraint development environment, 2006. Available from http://www.gecode.org. 
9. L. Getoor and B. Taskar, editors. An Introduction to Statistical Relational Learning. MIT Press, 2007.

10. Tias Guns, Siegfried Nijssen, and Luc De Raedt. k-pattern set mining under constraints. Technical Report CW 596, Department of Computer Science, Katholieke Universiteit Leuven, 2010.

11. Pascal Van Hentenreyck and Laurent Michel. Constraint-based Local Search. The MIT PRess, 2005.

12. Zhenjiang Hu, editor. Programming Languages and Systems, 7th Asian Symposium, APLAS 2009, Seoul, Korea, December 14-16, 2009. Proceedings, volume 5904 of Lecture Notes in Computer Science. Springer, 2009.

13. T. Imielinski and H. Mannila. A database perspectivce on knowledge discovery. Communications of the ACM, 39(11):58-64, 1996.

14. H. Mannila and H. Toivonen. Levelwise search and borders of theories in knowledge discovery. Data Mining and Knowledge Discovery, 1(3):241-258, 1997.

15. Tom Mitchell. The discipline of machine learning. Technical Report CMU-ML-06108, Carnegie Mellon University, 2006.

16. S. Muggleton and L. De Raedt. Inductive logic programming: Theory and methods. Journal of Logic Programming, 19/20:629-679, 1994.

17. Siegfried Nijssen, Tias Guns, and Luc De Raedt. Correlated itemset mining in ROC space: a constraint programming approach. In Proceedings of the 15th ACM SIGKDD International Conference on Knowledge Discovery and Data Mining, pages $647-656,2009$.

18. Luc De Raedt, Tias Guns, and Siegfried Nijssen. Constraint programming for itemset mining. In Proceedings of the 14th ACM SIGKDD International Conference on Knowledge Discovery and Data Mining, Las Vegas, Nevada, USA, August 2427, 2008, pages 204-212, 2008.

19. Francesca Rossi, Peter van Beek, and Toby Walsh. Handbook of Constraint Programming (Foundations of Artificial Intelligence). Elsevier Science Inc., 2006.

20. Christopher Simpkins, Sooraj Bhat, Charles Lee Isbell Jr., and Michael Mateas. Towards adaptive programming: integrating reinforcement learning into a programming language. In Gail E. Harris, editor, Proceedings of the 23rd Annual ACM SIGPLAN Conference on Object-Oriented Programming, Systems, Languages, and Applications, pages 603-614, 2008.

21. S. Sonnenburg, M.L. Braun, C.S. Ong, S. Bengio, L. Bottou, G. Holmes, Y. LeCun, K.-R. Müller, F. Pereira, C. A. Rasmussen, G. Rätsch, B. Schölkopf, A. Smola, P. Vincent, J. Weston, and R. Williamson. The need for open source software in machine learning. Journal of Machine Learning Research, 8:2443-2466, 2007. 